\title{
\begin{tabular}{l|l} 
Mibraries & DSpace@MIT
\end{tabular}
}

\author{
MIT Open Access Articles
}

\section{Gravitational-wave confusion background from cosmological compact binaries: Implications for future terrestrial detectors}

The MIT Faculty has made this article openly available. Please share how this access benefits you. Your story matters.

Citation: Regimbau, T. , and Scott A. Hughes. “Gravitational-wave confusion background from cosmological compact binaries: Implications for future terrestrial detectors." Physical Review D 79.6 (2009): 062002. (C) 2010 The American Physical Society.

As Published: http://dx.doi.org/10.1103/PhysRevD.79.062002

Publisher: American Physical Society

Persistent URL: http://hdl.handle.net/1721.1/51327

Version: Final published version: final published article, as it appeared in a journal, conference proceedings, or other formally published context

Terms of Use: Article is made available in accordance with the publisher's policy and may be subject to US copyright law. Please refer to the publisher's site for terms of use. 


\title{
Gravitational-wave confusion background from cosmological compact binaries: Implications for future terrestrial detectors
}

\author{
T. Regimbau* \\ UMR ARTEMIS, CNRS, University of Nice Sophia-Antipolis, Observatoire de la Côte d'Azur, BP 4229, 06304 Nice, France ${ }^{\dagger}$ \\ Scott A. Hughes \\ Department of Physics and MIT Kavli Institute, 77 Massachusetts Avenue, Cambridge, Massachusetts 02139, USA
}

(Received 19 January 2009; published 25 March 2009)

\begin{abstract}
Increasing the sensitivity of a gravitational-wave (GW) detector improves our ability to measure the characteristics of detected sources. It also increases the number of weak signals that contribute to the data. Because GW detectors have nearly all-sky sensitivity, they can be subject to a confusion limit: Many sources which cannot be distinguished may be measured simultaneously, defining a stochastic noise floor to the sensitivity. For GW detectors operating at present and for their planned upgrades, the projected event rate is sufficiently low that we are far from the confusion-limited regime. However, some detectors currently under discussion may have large enough reach to binary inspiral that they enter the confusionlimited regime. In this paper, we examine the binary inspiral confusion limit for terrestrial detectors. We consider a broad range of inspiral rates in the literature, several planned advanced gravitational-wave detectors, and the highly advanced "Einstein telescope" design. Though most advanced detectors will not be impacted by this limit, the Einstein telescope with a very low-frequency "seismic wall" may be subject to confusion noise. At a minimum, careful data analysis will be require to separate signals which will appear confused. This result should be borne in mind when designing highly advanced future instruments.
\end{abstract}

DOI: 10.1103/PhysRevD.79.062002

PACS numbers: 04.80.Nn, 04.30.Db, 04.30.Tv

\section{INTRODUCTION}

Compact binary coalescences, the gravitational-wave (GW) driven inspiral and merger of binaries whose members are neutron stars and/or black holes, are among the most promising sources of GWs for ground-based detectors. The late inspiral and merger waves from these binaries are very strong, and their waveforms are predictable. Their detection would open a direct window into strong gravitational dynamics, teaching us much about the evolution of massive stellar systems, the nature of gravity in the strong-field regime, and perhaps the nature of matter in neutron stars. The well-modeled nature of their waves may allow them to be used as standard candles (or standard sirens) $[1-5]$ to probe cosmology. By combining GW measurements, which encode distance to the source, with optical or radio observations of the host galaxy (for example, if the event is associated with a gamma-ray burst), which provides redshift, one can calibrate the relation between luminosity distance and redshift (the Hubble diagram) [6].

Such science goals have played a large role in driving plans for ever more sensitive GW detectors. Improved GW sensitivity impacts the measurement of binary coalescence events in two ways. First, any measured event is characterized with greater precision by an instrument with high

\footnotetext{
*regimbau@oca.eu

${ }^{\dagger}$ URL: http://www.oca.eu/regimbau
}

sensitivity thanks to improved signal-to-noise ratio. Many of the analyses of GW science from compact binary measurement assume measurement by "advanced" detectors (e.g., $[7,8]$ ), with sensitivity to GWs roughly a factor of 10 or so greater than those in operation today. Second, the "detection horizon" to which events can be measured grows as sensitivity is improved. GW detector noise is normally quoted as a strain sensitivity. Doubling an instrument's sensitivity increases its detection horizon by a factor of 2, and hence the volume to which an instrument is sensitive by a factor of 8 . The rate at which events are measured can in principle be substantially increased by relatively modest improvements in instrumental sensitivity.

Such improvement in sensitivity is on the one hand mandated by the rarity of strong gravitational-wave events. Extrapolation from the observed binary pulsar systems in our galaxy and population synthesis calculations each suggest that binary mergers occur roughly once every hundred thousand years per Milky Way equivalent galaxy; a more precise discussion of the merger rate is given in Sec. II below. Combining this rate with the density of galaxies in our universe drives us to the need for a detection horizon of a few hundred Mpc in order to measure multiple neutron star-neutron star (NS-NS) events per year. On the other hand, there can be too much of a good thing: If the detection horizon is sufficiently far out, sources may be detected so often that they overlap, making a transition to a confusion-limited background. The transition from dis- 
crete sources to confused background has been discussed extensively, especially in the context of sources for the space-based detector LISA $[9,10]$.

In this paper, we investigate the threshold redshift at which merging binary sources for ground-based detectors begin to overlap and result in a confusion background. Beyond this redshift, it will become difficult to resolve sources individually. As has been found in the LISA Mock Data Challenges, separation may be possible by globally fitting for all sources simultaneously (see, e.g., Ref. [11]); similar analyses have been done for the proposed highly advanced Big Bang Observatory [12]. Careful analysis will be needed to see how well such techniques can be applied to ground-based detector data. At any rate, it is likely that improving sensitivity to probe beyond this point will not be worthwhile. We first discuss estimates of the cosmic coalescence rates of NS-NS and neutron star-black hole (NS$\mathrm{BH})$ systems in Sec. II. In Sec. III, we then discuss the different detection regimes which pertain to binary coalescence measurement. Key to this discussion is understanding how long different sources are in band, which depends most strongly on the low-frequency sensitivity of the instrument being used to measure the waves. We consider the initial and planned advanced LIGO and Virgo detectors and three possible configurations of the Einstein telescope concept. Our conclusions are given in Sec. IV. Chief among them is that confusion issues are likely to become important for ground-based GW detectors which are sensitive to these events to a redshift $z \sim 1$, especially if such instruments have a "seismic wall" at or below roughly $5 \mathrm{~Hz}$. This may have important ramifications for designing highly sensitive future detectors such as the Einstein telescope. At a minimum, it will be necessary to carefully design the data analysis to disentangle these potentially confused sources.

\section{COALESCENCE RATES}

To motivate the choices for our calculations, we begin by reviewing our current understanding of the rate of NSNS and NS-BH coalescences in the universe. The final merger of a compact binary occurs after two massive stars in a binary system have collapsed to form neutron stars or black holes ${ }^{1}$ and have inspiralled through the emission of gravitational waves. We assume that the coalescence rate tracks the star formation rate, albeit with some delay $t_{d}$ from formation of the binary to final merger. Accordingly, we put

$$
\dot{\rho}_{c}^{\mathrm{o}}(z)=\dot{\rho}_{c}^{\mathrm{o}}(0) \times \frac{\dot{\rho}_{*, c}(z)}{\dot{\rho}_{*, c}(0)} .
$$

In Eq. (1), $\dot{\rho}_{c}^{\mathrm{o}}(z)$ represents the rate at which binary systems are observed to merge at redshift $z$, and $\dot{\rho}_{c}^{\mathrm{o}}(0)$ is this rate in

\footnotetext{
${ }^{1} \mathrm{We}$ neglect the possible production of compact binaries through interactions in dense star systems.
}

our local universe. This normalization reproduces the local rate for $z=0$. We review estimates of $\dot{\rho}_{c}^{\mathrm{o}}(0)$ in more detail later in this section. The quantity $\dot{\rho}_{*, c}$ relates the past star formation rate (SFR) to the rate of binary merger. It is defined as

$$
\dot{\rho}_{*, c}(z)=\int \frac{\dot{\rho}_{*}\left(z_{f}\right)}{\left(1+z_{f}\right)} P\left(t_{d}\right) d t_{d}
$$

In Eq. (2), $\dot{\rho}_{*}$ is the SFR, measured in $\mathrm{M}_{\odot} \mathrm{Mpc}^{-3} \mathrm{yr}^{-1}$. The redshift $z$ describes when our compact binary merges, and $z_{f}$ is the redshift at which its progenitor binary formed. These redshifts are connected by the time delay $t_{d}$, which is the sum of the time from initial binary formation to evolution into compact binary, plus the merging time $\tau_{m}$ by emission of gravitational waves. It is also the difference in lookback times between $z_{f}$ and $z$ :

$$
t_{d}=t_{\mathrm{LB}}\left(z_{f}\right)-t_{\mathrm{LB}}(z)=\frac{1}{H_{0}} \int_{z}^{z_{f}} \frac{d z^{\prime}}{\left(1+z^{\prime}\right) E\left(\Omega, z^{\prime}\right)},
$$

where

$$
E(\Omega, z)=\sqrt{\Omega_{\Lambda}+\Omega_{m}(1+z)^{3}} .
$$

We use the 737 cosmology [13], with $\Omega_{m}=0.3, \Omega_{\Lambda}=$ 0.7 , and Hubble parameter $H_{0}=70 \mathrm{~km} \mathrm{~s}^{-1} \mathrm{Mpc}^{-1}$ (or equivalently $h_{0}=0.7$ ). This corresponds to the concordance model derived from observations of distant type Ia supernovae [14] and the power spectrum of the cosmic microwave background fluctuations [15]. Finally, in Eq. (2) $P\left(t_{d}\right)$ is the probability distribution for the delay $t_{d}$. The factor $1 /\left(1+z_{f}\right)$ accounts for time dilatation due to the cosmic expansion.

We find the merger rate per unit redshift by multiplying $\dot{\rho}_{c}^{\mathrm{o}}(z)$ with the element of comoving volume:

$$
\frac{d R_{c}^{\mathrm{o}}}{d z}(z)=\dot{\rho}_{c}^{\mathrm{o}}(z) \frac{d V}{d z}(z)
$$

where

$$
\begin{gathered}
\frac{d V}{d z}(z)=4 \pi \frac{c}{H_{0}} \frac{r(z)^{2}}{E(\Omega, z)}, \\
r(z)=\frac{c}{H_{0}} \int_{0}^{z} \frac{d z^{\prime}}{E\left(\Omega, z^{\prime}\right)} .
\end{gathered}
$$

Note that Eq. (7) assumes spatial flatness, $\Omega_{m}+\Omega_{\Lambda}=1$.

For $\dot{\rho}_{*}$, we use the recent SFR of [16], which is derived from new measurements of the galaxy luminosity function in the UV (SDSS, GALEX, COMBO17) and FIR wavelengths (Spitzer Space Telescope), and is normalized by the Super Kamiokande limit on the electron antineutrino flux from past core-collapse supernovas. This model is expected to be quite accurate up to $z \sim 2$, with very tight constraints at redshifts $z<1$ (to within 30\%-50\%). To account for uncertainty in reconstructing the SFR, we also considered the model described in Fardal et al. (Ref. [17]). That work uses a different set of measurements 


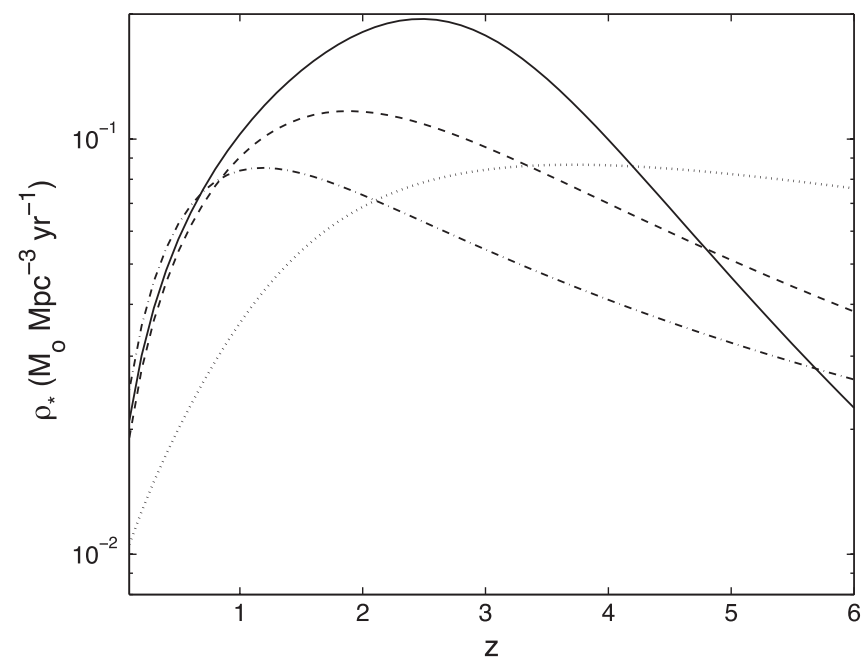

FIG. 1. Cosmic star formation rates (in $\mathrm{M}_{\odot} \mathrm{Mpc}^{-3} \mathrm{yr}^{-1}$ ) used in this paper: Hopkins and Beacom 2006 [16] (continuous line), Fardal et al. 2007 [17] (dashed line), Wilkins et al. 2008 (dotdashed line) [18], and the fossil model of Nagamine et al. 2006 (dotted line). As discussed in the text, these rates are largely the same up to $z \sim 1$ but show important differences at higher redshift.

and a different dust extinction correction. The SFR found in [17] is the same as that of [16] up to $z \sim 1$, but decreases slightly at higher redshifts. We also consider the model described by Wilkins et al. in Ref. [18], which is derived from measurements of the stellar mass density. The SFR is equivalent to that in $[16,17]$ for $z \lessgtr 0.7$, but again is lower at higher redshifts. Finally, we consider the SFR of Ref. [19], which is derived from the fossil record of star formation in nearby galaxies. It is probably underestimated at small redshifts and is constant at high redshifts due to the contribution of elliptical galaxies. Note that at present there is a discrepancy between the "instantaneous" SFR, measured from the emission of young stars in star forming regions, and the SFR as determined from extragalactic background light. This could have an important impact on the contribution to the confusion background for sources from $z>2$. The star formation rates which we use are summarized in Fig. 1.

Population synthesis [20-26] suggests that the delay time $t_{d}$ is well described by a probability distribution of the form

$$
P_{d}\left(t_{d}\right) \propto \frac{1}{t_{d}} \quad \text { with } \quad t_{d}>\tau_{0}
$$

for some minimal delay time $\tau_{0}$. This broad model accounts for the wide range of merger times observed in binary pulsars; it is also consistent with short gamma-ray burst observations in both late and early-type galaxies [27]. Following [26,28], who identify a channel which produces tight NS-NS binaries with merger time in the range $\tau_{m} \sim$ 0.001-0.1 Myr, we assume a minimal delay time for NSNS of $\tau_{0} \sim 20 \mathrm{Myr}$. This corresponds roughly to the time it takes for massive binaries to evolve into two neutron stars. For NS-BHs, we take a minimal delay of $\tau_{0} \sim$ $100 \mathrm{Myr}$ [29], corresponding to the wider orbits and longer merger times predicted by classical channels. Figure 2 shows the cosmic coalescence rate as a function of redshift, normalized to the local value, which results from this model.

The local merger rate per unit volume, $\dot{\rho}_{c}^{\mathrm{o}}(0)$, is usually extrapolated by multiplying the rate in the Milky Way $\left(r_{\mathrm{mw}}\right)$ with the density of Milky-Way equivalent galaxies. That density, in turn, is found by measurements of the blue stellar luminosity to be roughly $n_{\mathrm{mw}} \sim(1-2) \times$ $10^{-2} \mathrm{Mpc}^{-3}$ [30-32]. Current estimates of the NS-NS galactic coalescence rate which extrapolate from observed galactic NS-NS find a rate in the range 17-292 $\mathrm{Myr}^{-1}$ (95\% confidence interval), with a most likely rate of $83 \mathrm{Myr}^{-1}$ [33]. Population synthesis predicts a NS-NS merger rate in the range $1-300 \mathrm{Myr}^{-1}$ (most likely near 10-30 $\mathrm{Myr}^{-1}$; see Table I for a summary). Rate estimates for NS-BH systems are one or two orders of magnitude smaller, ranging between $0.1-30 \mathrm{Myr}^{-1}$ (Table I).

It is worth noting that the stellar mass fraction which produces massive binaries is expected to be larger in earlytype galaxies than in spiral galaxies thanks to their flatter initial mass function. Despite their absence of recent star formation, early-type galaxies may make a larger contribution to the merger rate than spiral galaxies due to binaries with very long coalescence times which were born in a galaxy's first 1-2 Gyr. Assuming that ellipticals represent a fraction of about $40 \%$ of the galaxies, [23] find a small correction factor $\sim 2$.

TABLE I. Current estimates of the Galactic merger rates of NS-NSs and NS-BHs in units of $\mathrm{Myr}^{-1}$, adapted from Table 4 of [34]. The rates from Ref. [33] are derived from statistical studies; the numbers in parenthesis give the $95 \%$ confidence limits around the maximum likelihood value. All other values are estimated using population synthesis. The high rate obtained in Ref. [35] is due to the assumption that neutron stars or black holes are born with no kick velocity, leading to an overestimate of the number of systems that survive both supernovae. The low rate obtained by Ref. [36] is due to their treatment of common envelope binding physics.

\begin{tabular}{lll}
\hline \hline Statistics & NS-NS \\
Kalogera et al. (2004) [33] & 83 (17-292) \\
\hline Population synthesis & NS-NS & NS-BH \\
Tutunov and Yungelson (1993) [35] & 300 & 20 \\
Lipunov et al. (1997) [37] & 30 & 2 \\
Portegies Zwart and Yungelson (1998) [38] 20 & 2 \\
Nelemans et al. (2001) [39] & 20 & 4 \\
Voss and Tauris (2003) [36] & 2 & 0.6 \\
de Freitas Pacheco et al. (2006) [23] & 17 & \\
Belczinsky et al. (2007) [40] & $10-15$ & 0.1 \\
O'Shaughnessy et al. (2008) [22] & 30 & 3 \\
\hline \hline
\end{tabular}


Taking these uncertainties into account, we consider local rates in the range $\dot{\rho}_{c}^{\mathrm{o}}(z=0)=(0.01-10) \times$ $\mathrm{Myr}^{-1} \mathrm{Mpc}^{-3}$ for NS-NS and $\dot{\rho}_{c}^{\mathrm{o}}(z=0)=$ (0.001-1) $\mathrm{Myr}^{-1} \mathrm{Mpc}^{-3}$ for NS-BH. Our two reference models for NS-NS are

(i) $\dot{\rho}_{c}^{\mathrm{o}}(z=0) \sim 1 \mathrm{Myr}^{-1} \mathrm{Mpc}^{-3}$, corresponding to the most probable galactic rate of $r_{\mathrm{mw}}=83 \mathrm{Myr}^{-1}$, and

(ii) $\dot{\rho}_{c}^{\mathrm{o}}(z=0) \sim 0.4 \mathrm{Myr}^{-1} \mathrm{Mpc}^{-3}$, corresponding to the prediction of the latest population synthesis of [22] $\left(r_{\mathrm{mw}}=30 \mathrm{Myr}^{-1}\right.$ or to $r_{\mathrm{mw}}=15 \mathrm{Myr}^{-1}$ $[23,40]$, with a correction factor of 2 due to ellipticals).

Our reference model for NS-BH is $\dot{\rho}_{c}^{\mathrm{o}}(z=0) \sim$ $0.04 \mathrm{Myr}^{-1} \mathrm{Mpc}^{-3}$, corresponding to the most recent predictions of [41]. For all three models, we assume $n_{\mathrm{mw}}=$ $1.2 \times 10^{-2} \mathrm{Mpc}^{-3}[32]$.

\section{DETECTION REGIMES}

Turn now to the measurement of signals from NS-NS and NS-BH binaries. The contribution of these binaries to the instrumental data falls into three statistically very different regimes, depending mostly on the typical interval between events (see [42,43] and references within):

(1) Shot noise: This case describes when the number of sources is small enough that the interval between events is long compared to an individual event's duration. Measured waves are separated by long stretches of silence and can be resolved individually. This case pertains to instruments that are only sensitive to events at low redshift.

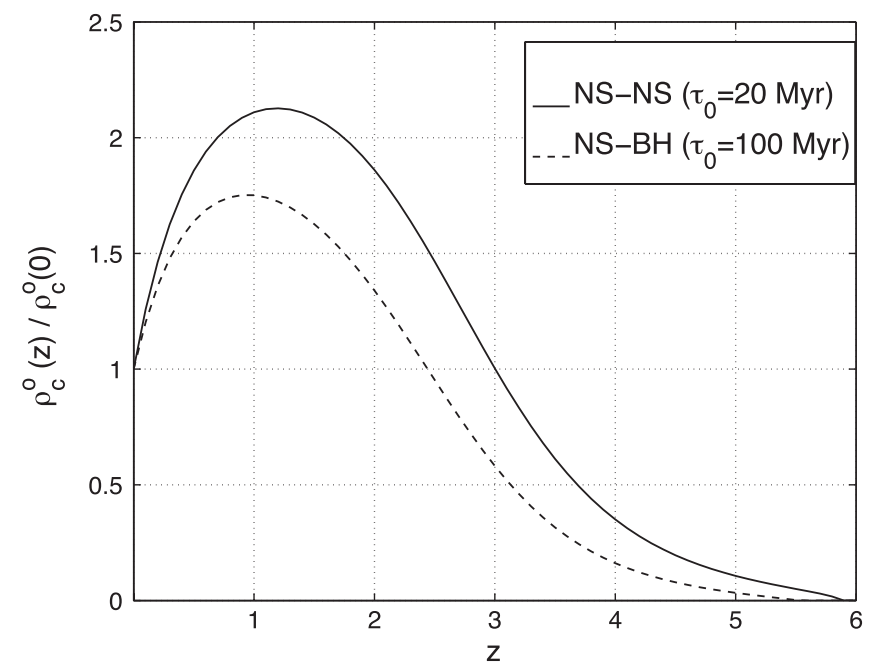

FIG. 2. Cosmic coalescence rate, normalized to the local value $\dot{\rho}_{c}^{\mathrm{o}}(z=0)$, for models with a distribution of the delay of the form $P\left(t_{d}\right) \propto 1 / t_{d}$. The continuous line is the distribution for a minimal delay $\tau_{0}=20 \mathrm{Myr}$, which is assumed to be representative for NS-NS binaries. The dashed line is for a delay of $100 \mathrm{Myr}$, taken to be representative of NS-BHs. In both cases, we have assumed the cosmic star formation rate of Hopkins and Beacom [16].
(2) Popcorn noise: As the reach of instruments increases, the time interval between events may come closer to the duration of single bursts. Events may sometimes overlap, making it difficult to distinguish between them.

(3) Continuous: For instruments with very large reach and excellent low-frequency sensitivity, the interval between events can be small compared to the duration of an event. The signals overlap to create a confusion noise of unresolved sources.

The average number of measured events at a given moment is given by the duty cycle $\Delta$. It is defined as the ratio, in the observer frame, of the typical duration of a single event $\tau$, to the average time interval between successive events

$$
\Delta(z)=\int_{0}^{z} \tau\left(z^{\prime}\right) \frac{d R_{c}^{\mathrm{o}}}{d z^{\prime}}\left(z^{\prime}\right) d z^{\prime}
$$

Here, $d R_{c}^{\mathrm{o}} / d z^{\prime} \propto \dot{\rho}_{c}^{\mathrm{o}}$ is the coalescence rate per unit redshift, given by Eq. (5); $\tau\left(z^{\prime}\right)$ is the observed duration of a GW signal generated at redshift $z^{\prime}$. This duration is given at leading order by

$$
\tau\left(z^{\prime}\right)=\frac{5 c^{5}}{256 \pi^{8 / 3} G^{5 / 3}}\left[\left(1+z^{\prime}\right) m_{c}\right]^{-5 / 3} f_{L}^{-8 / 3},
$$

where $m_{c}=\left(m_{1} m_{2}\right)^{3 / 5} /\left(m_{1}+m^{2}\right)^{1 / 5}$ is the binary's chirp mass, and where $f_{L}$ is the lower frequency bound of the detector, assumed to be much smaller than the frequency at the time of the merger.

The signal duration depends very strongly on both chirp mass and lower frequency bound; in particular, signals measured by instruments with very low $f_{L}$ may have very long durations. We will take typical NS masses to be $1.4 \mathrm{M}_{\odot}$ and typical $\mathrm{BH}$ mass to be $9.5 \mathrm{M}_{\odot}$, yielding chirp masses $m_{c}=1.2 \mathrm{M}_{\odot}$ for NS-NS and $m_{c}=2.9 \mathrm{M}_{\odot}$ for NS-BH. These values agree well with the most probable values derived by [40] with the StarTrack population synthesis code, for their reference model. The lower frequency bound is determined by the properties of the detector used for the measurement. Sensitivity curves describing the instruments we include in our analysis are shown in Fig. 3. We consider:

(i) Initial LIGO: The present LIGO interferometers [44] have a low-frequency seismic "wall" at roughly $40 \mathrm{~Hz}$. NS-NS binaries are in band for roughly 25 seconds; NS-BH binaries are in band for roughly 5.8 seconds. The isotropic detection horizon (the angle-averaged distance to which a binary can be measured, defined carefully below) is at a luminosity distance of $15 \mathrm{Mpc}(z=0.0035)$ for NS-NS and $30 \mathrm{Mpc}$ for NS-BH $(z=0.007)$ for the LIGO Hanford $4 \mathrm{~km}$ detector. When both $4 \mathrm{~km}$ detectors (at Hanford, Washington and Livingston, Louisiana) and the single $2 \mathrm{~km}$ detector (at Hanford) are com- 
bined (assuming independence of all detectors and stationary noise), these numbers increase by a factor of about $\sqrt{1^{2}+1^{2}+(1 / 2)^{2}}=1.5$ to $22 \mathrm{Mpc}(z=$ $0.005)$ for NS-NS and $44 \mathrm{Mpc}$ for NS-BH $(z=$ $0.01)$.

(ii) Virgo: The Virgo design sensitivity [45] is comparable to the LIGO sensitivity, but with advanced seismic isolation provided by the so-called "superattenuator" (see, e.g., Ref. [46] for discussion). A low-frequency wall at $10 \mathrm{~Hz}$ means that NS-NS signals will be in band for 16.7 minutes; NS-BH signals last for 3.9 minutes. The Virgo sensitivity is slightly poorer than the LIGO sensitivity between $100-300 \mathrm{~Hz}$, so its isotropic detection horizon is $11 \mathrm{Mpc}(z=0.0025)$ for NS-NS and $23 \mathrm{Mpc}$ for NS-BH $(z=0.0055)$.

(iii) Advanced LIGO/VIRGO: The advanced LIGO design $[47,48]$ should move its wall down to a frequency of roughly $10 \mathrm{~Hz}$. NS-NS signals will be in band for about 16.7 minutes and have a single detector detection horizon of $200 \mathrm{Mpc}(z=0.045)$; NS-BH signals will be in band for 3.9 minutes and have a detection horizon of $420 \mathrm{Mpc}(z=0.09)$. The current advanced LIGO design plan will have three $4 \mathrm{~km}$ instruments; combining their data brings the isotropic horizon to $355 \mathrm{Mpc}(z=0.08)$ for NS-NS and $765 \mathrm{Mpc}(z=0.16)$ for NS-BH. The plans for advanced Virgo sensitivity [49] are comparable to those for LIGO. The expected isotropic detection horizon for advanced Virgo is at $150 \mathrm{Mpc}(z=$ $0.035)$ for NS-NS and $310 \mathrm{Mpc}$ for NS-BH $(z=$ $0.07)$.

(iv) The Einstein telescope: Several possible Einstein telescope designs are presently under discussion. The plan is to move the seismic wall down to a frequency below $5 \mathrm{~Hz}$. A planned low-frequency wall at $5 \mathrm{~Hz}$ would mean that NS-NS signals will be in band for 1.8 hours; NS-BH signals will be for 24.6 minutes. Another possibility will be to lower it to $3 \mathrm{~Hz}$ (so that NS-NS signals are in band for 6.9 hours and NS-BH for 1.6 hours), or even to $1 \mathrm{~Hz}$ (NS-NS lasting for 5.4 days and NS-BH for 1.2 days). The Einstein telescope detection horizon for NS-NS signals is at $z \simeq 1$; for NS-BH, it is at $z \simeq$ 2.

The isotropic detection horizon for these instruments is the distance at which the angle-averaged signal-to-noise ratio $\rho$, defined by

$$
\rho^{2}=4\left\langle\int \frac{|\tilde{h}(f)|^{2}}{h_{n}(f)^{2}} d f\right\rangle,
$$

equals the threshold value $\rho_{\text {th }}=8$. In Eq. (11), $\tilde{h}(f)$ is the Fourier transform of the inspiral waveform, $h_{n}(f)$ is the strain noise (in units of $\mathrm{Hz}^{-1 / 2}$, as shown in Fig. 3), and \langle\rangle

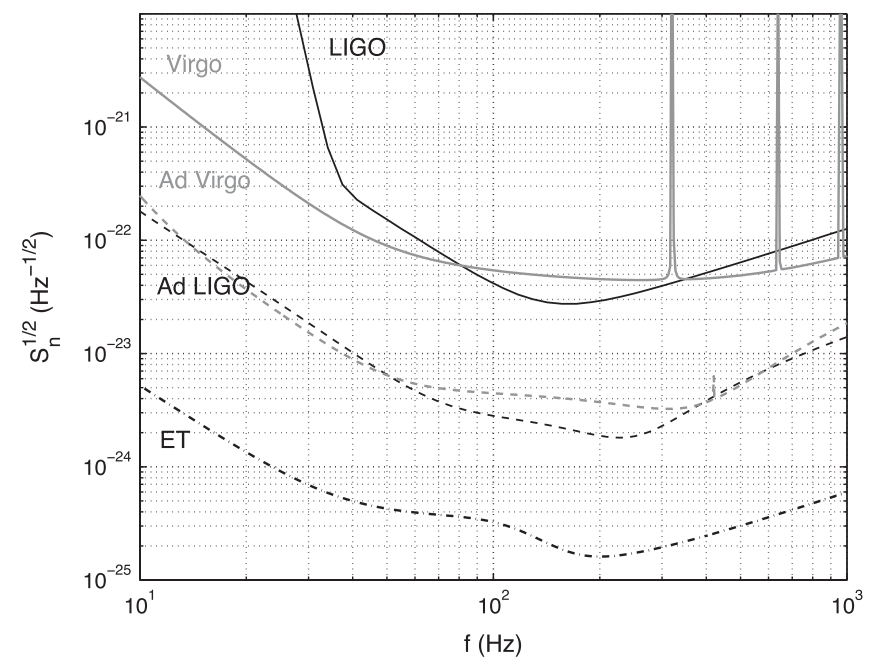

FIG. 3. Strain sensitivities, in $\mathrm{Hz}^{-1 / 2}$, for the instruments that we consider as functions of frequency: LIGO $4 \mathrm{~km}$ as specified in the Science Requirement document [44]; design initial Virgo [45]; planned advanced LIGO and Virgo [47-49] (optimized for NS-NS detection); and a possible Einstein telescope design (Lshaped, $10 \mathrm{~km}$ ) [ET science team, private communication].

denotes an appropriate angle averaging; see, for example, Ref. [6], Sec. II for discussion. We are careful to specify the "isotropic" detection horizon in our discussion (in reference to this angle averaging) to contrast with the term "detection horizon." This name is typically used to denote the distance to which an optimally oriented and position source could be detected. Whereas fortunate orientation and position may make a source visible beyond the isotropic detection horizon, no source is visible beyond the detection horizon. The threshold $\rho_{\text {th }}=8$ is chosen to keep the false alarm rate (for Gaussian noise statistics) acceptably low.

The redshift $z_{*}$ at which sources start to overlap to produce a confusion background is the transition between the shot noise and the popcorn noise regimes. This is defined by the condition

$$
\Delta\left(z_{*}\right)=1
$$

In this regime, where the number of sources that overlap is small, it may still be possible to separate the sources individually with adequate data analysis techniques. We therefore consider in addition a more conservative threshold $z_{* *}$, corresponding to the transition between the popcorn regime and the Gaussian stochastic background. It is defined by $\Delta\left(z_{* * *}\right)=10$.

Figure 4 shows $\Delta(z)$ for different estimates of the local coalescence rate of NS-NSs and NS-BHs and for an instrumental seismic wall of $10 \mathrm{~Hz}$; Figs. 5 and 6 show the same thing for seismic walls at 5 and $1 \mathrm{~Hz}$, respectively. The threshold redshifts $z_{*}$ and $z_{* *}$ for NS-NS are shown in 

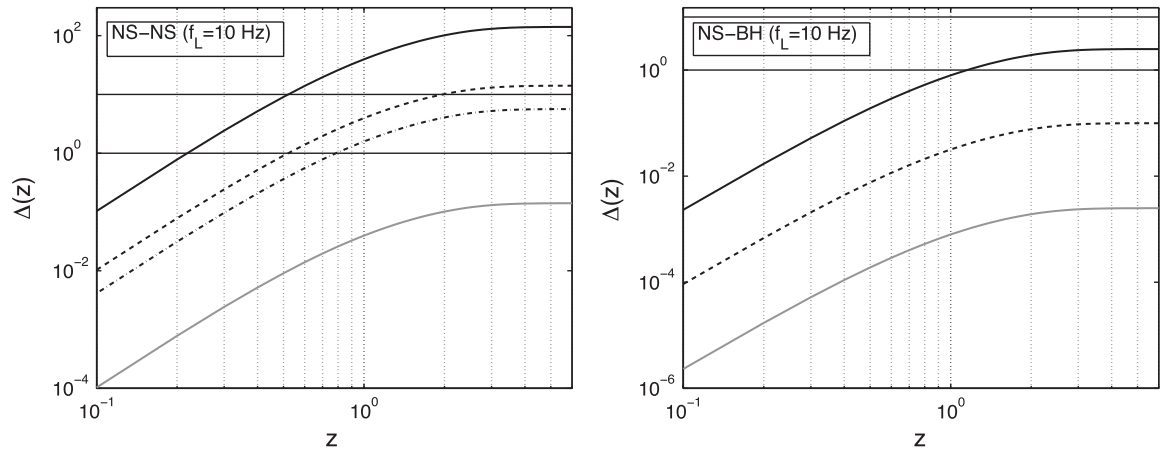

FIG. 4. Duty cycle as a function of redshift for NS-NSs (top) and NS-BHs (bottom), for a lower frequency bound of $10 \mathrm{~Hz}$, and for our reference SFR [16]. The continuous black and grey lines correspond to the most optimistic and pessimistic estimates of the local coalescence rate, respectively; the dashed and dot-dash lines correspond to our reference models (cf. Sec. II). The horizontal line at $\Delta(z)=1$ indicates the transition between resolved sources and a popcorn background; the line at $\Delta(z)=10$ is our more conservative transition to a confused background.
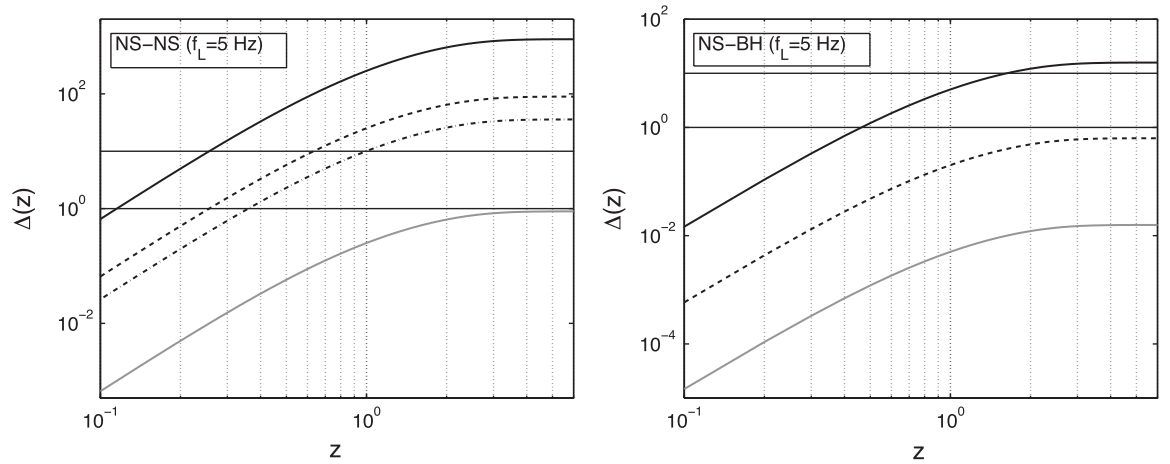

FIG. 5. Same as Fig. 4 for a lower frequency bound of $5 \mathrm{~Hz}$.
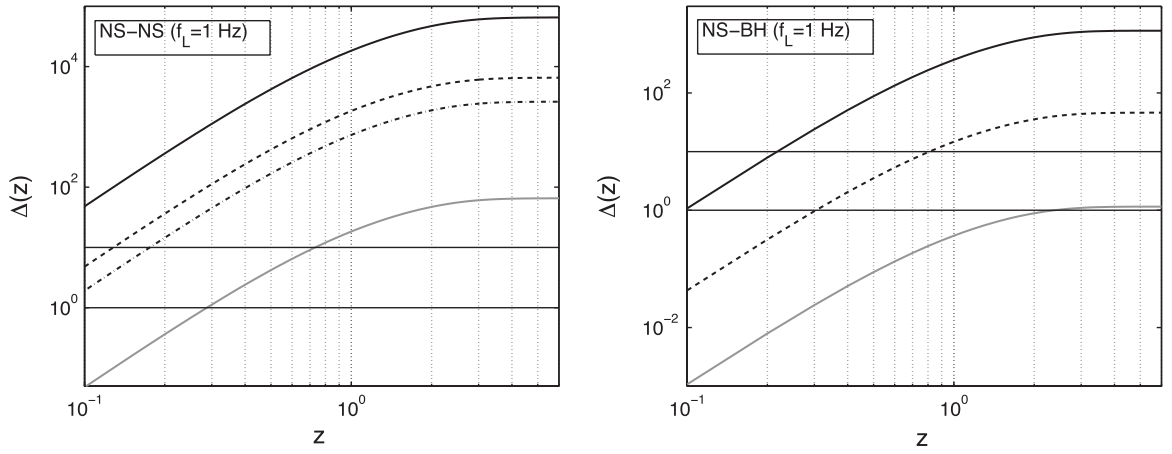

FIG. 6. Same as Fig. 4 for a lower frequency bound of $1 \mathrm{~Hz}$.

Table II; Table III shows these thresholds for NS-BH. For ease of comparison, we repeat in these tables the detection horizons for the various instruments discussed above.

\section{DISCUSSION AND CONCLUSIONS}

The main concern of the first and second generations of interferometric gravitational-wave detectors will be improving sensitivity to ensure unambiguous first detection of gravitational waves, followed by measurement with sufficient precision to inaugurate gravitational-wave astronomy as an observational science. As the technology improves and these instruments' sensitivity increases, the possibility emerges that a confusion background may become an important effect, ultimately limiting the capabilities of these instruments.

Referring to Tables II and III, we see that there is little danger of a confusion background impacting these instru- 
TABLE II. Top entries: threshold between resolved and unresolved NS-NS binaries for different estimates of the source rate $\dot{\rho}_{c}^{\mathrm{o}}$ and detector lower frequency bound $f_{L}$. No value means that the duty cycle is always $<1$ or $<10$. Bottom entries: lower frequency sensitivity bound and detection horizon for current and planned gravitational-wave detectors. For LIGO, the horizon is given for the SRD $4 \mathrm{~km}$ detector and the value in parenthesis corresponds to the combination of the three detectors.

\begin{tabular}{|c|c|c|c|}
\hline$\underline{f_{L}}$ & $\dot{\rho}_{c}^{\mathrm{o}}$ & $z_{*}$ & $z_{* *}$ \\
\hline \multirow[t]{4}{*}{10} & 0.01 & & $\ldots$ \\
\hline & 0.4 & $0.8-0.9$ & $\ldots$ \\
\hline & 1 & $0.5-0.6$ & $>2$ \\
\hline & 10 & 0.2 & $0.5-0.6$ \\
\hline \multirow[t]{4}{*}{5} & 0.01 & $\ldots$ & $\ldots$ \\
\hline & 0.4 & 0.4 & $1-1.2$ \\
\hline & 1 & 0.25 & $0.6-0.7$ \\
\hline & 10 & 0.1 & 0.25 \\
\hline \multirow[t]{4}{*}{1} & 0.01 & 0.3 & 0.8 \\
\hline & 0.4 & 0.08 & 0.2 \\
\hline & 1 & 0.06 & 0.13 \\
\hline & 10 & 0.03 & 0.06 \\
\hline \multicolumn{4}{|r|}{$z_{\mathrm{DH}}=0.0035(0.005)$} \\
\hline $\begin{array}{l}\text { LIGO: } \\
\text { Virgo: }\end{array}$ & & $f_{L}=10 \mathrm{~Hz}$ & $z_{\mathrm{DH}}=0.0025$ \\
\hline \multicolumn{2}{|c|}{ Advanced LIGO: } & $f_{L}=10 \mathrm{~Hz}$ & $z_{\mathrm{DH}}=0.045(0.08)$ \\
\hline \multicolumn{2}{|c|}{ Advanced Virgo: } & $f_{L}=5 \mathrm{~Hz}$ & $z_{\mathrm{DH}}=0.035$ \\
\hline \multicolumn{2}{|c|}{ Einstein telescope: } & $f_{L}=\{5,3,1\} \mathrm{Hz}$ & $z_{\mathrm{DH}} \simeq 1$ \\
\hline
\end{tabular}

ments even into the advanced LIGO era. The confusion background only has an impact when a detector's "reach" (summarized by the detection horizon $z_{\mathrm{DH}}$ ) becomes large and its low-frequency wall (the cutoff frequency $f_{L}$ ) be-

TABLE III. Same as Table. II, but for NS-BH binaries.

\begin{tabular}{|c|c|c|c|}
\hline$f_{L}$ & $\dot{\rho}_{c}^{\mathrm{o}}$ & $z_{*}$ & $z_{* *}$ \\
\hline \multirow[t]{3}{*}{10} & 0.001 & $\ldots$ & $\ldots$ \\
\hline & 0.04 & $\ldots$ & $\ldots$ \\
\hline & 1 & $1.1-1.4$ & $\ldots$ \\
\hline \multirow[t]{3}{*}{5} & 0.001 & $\ldots$ & $\ldots$ \\
\hline & 0.04 & $\ldots$ & $\ldots$ \\
\hline & 1 & 0.5 & $>1.6$ \\
\hline \multirow[t]{3}{*}{1} & 0.001 & $>2.3$ & $\ldots$ \\
\hline & 0.04 & 0.3 & $0.8-0.9$ \\
\hline & 1 & 0.1 & 0.2 \\
\hline & $f_{L}=40 \mathrm{~Hz}$ & $z_{\mathrm{DH}}=0.007(0.01)$ \\
\hline \multicolumn{2}{|c|}{ Virgo: } & $f_{L}=10 \mathrm{~Hz}$ & $z_{\mathrm{DH}}=0.0055$ \\
\hline \multicolumn{2}{|c|}{ Advanced LIGO: } & $f_{L}=10 \mathrm{~Hz}$ & $z_{\mathrm{DH}}=0.09(0.16)$ \\
\hline \multicolumn{2}{|c|}{ Advanced Virgo: } & $f_{L}=5 \mathrm{~Hz}$ & $z_{\mathrm{DH}}=0.07$ \\
\hline \multicolumn{2}{|c|}{ Einstein telescope: } & $f_{L}=\{5,3,1\} \mathrm{Hz}$ & $z_{\mathrm{DH}} \simeq 2$ \\
\hline
\end{tabular}

comes low. A distant detection horizon can greatly increase the number of sources that an instrument can reach; a lowfrequency wall can greatly increase the amount of time that a source spends in band.

The combination of these two effects is summarized by the duty cycle, $\Delta(z)$, which is the ratio of the typical duration of a measured event to the typical interval between events. We find that, for advanced detectors with excellent low-frequency sensitivity (such as the planned advanced Virgo design), the redshift $z_{*}$ at which the duty cycle for NS-NS is unity, $\Delta\left(z_{*}\right)=1$, may occur close to the detection horizon for the reference coalescence rate. The redshift $z_{*}$ defines a "popcorn" background in the language of Sec. III. The conservative redshift $z_{* *}$ for which $\Delta\left(z_{* * *}\right)=10$ (defining a Gaussian stochastic confusion background) may even be close to the detection horizon for optimistic event rates.

We find that, for NS-NS, both $z_{*}$ and $z_{* *}$ are well within the horizon of the planned Einstein telescope, if its lowfrequency sensitivity is at $f_{L}=1 \mathrm{~Hz}$ (ET1). If $f_{L}=5 \mathrm{~Hz}$ (ET5), we expect the popcorn background to occur before the detection horizon and more likely around $z_{*} \sim$ $0.25-0.4$, unless our most pessimistic coalescence rates are accurate $\left(\dot{\rho}_{c}^{\mathrm{o}}<0.015 \mathrm{Myr}^{-1} \mathrm{Mpc}^{-3}\right)$. The transition to a Gaussian stochastic most likely occurs at $z_{* *} \sim$ $0.6-1.2$, but can fall beyond the detection horizon if $\dot{\rho}_{c}^{\mathrm{o}}<$ $0.15 \mathrm{Myr}^{-1} \mathrm{Mpc}^{-3}$.

Our conclusions for NS-BH binaries are similar to those for NS-NS for ET1, $z_{*}$ and $z_{* *}$ are both more likely to occur well below the horizon. For ET5, however, there is not likely to be enough sources to create a Gaussian stochastic background (even a popcorn background), except for the most optimistic coalescence rates $\left(\dot{\rho}_{c}^{\mathrm{o}}>\right.$ $\left.0.6 \mathrm{Myr}^{-1} \mathrm{Mpc}^{-3}\right)$. As a consequence, NS-BHs may always be resolved provided that we can separate them with adequate data analysis strategies in the popcorn regime.

This result motivates very careful analysis of how data from an Einstein telescope-type instrument would be analyzed. Experience from the Mock LISA Data Challenges [11] and ideas developed for the Big Bang Observatory [12] prove that disentangling multiple signals in a gravitational-wave detector's datastream is certainly possible. However, beyond the proof of the concept, it is not clear how many of the lessons from these examples carry over to the case of ground-based detectors. In particular, even for an Einstein telescope-type detector, the bulk of the potentially confused signals would be of low signal-tonoise ratio since most events will come from the volume of the universe near the detection horizon. It would be a valuable exercise to examine how well techniques that simultaneously fit multiple signals do in the ET regime.

\section{ACKNOWLEDGMENTS}

We thank B. Sathyaprakash, C. van den Broeck, and D. McKechan for providing information about the Einstein 
telescope, D. Shoemaker, A. Vicere, and G. Losurdo for helpful discussions about advanced LIGO and Virgo, and I. Mandel for sharing a useful document on the coalescence rates. S.A.H. is supported by NSF Grant No. PHY-
0449884 and by NASA Grant No. NNX08AL42G, and gratefully acknowledges support from the MIT Class of 1956 Career Development Fund.
[1] B. F. Schutz, Nature (London) 323, 310 (1986).

[2] D. F. Chernoff and L. S. Finn, Astrophys. J. Lett. 411, L5 (1993).

[3] L. S. Finn, Phys. Rev. D 53, 2878 (1996).

[4] D. E. Holz and S. A. Hughes, Astrophys. J. 629, 15 (2005).

[5] K. Arun, B. R. Iyer, B. S. Sathyaprakash, S. Sinha, and C. van den Broeck, Phys. Rev. D 76, 104016 (2007).

[6] N. Dalal, D. E. Holz, S. A. Hughes, and B. Jain, Phys. Rev. D 74, 063006 (2006).

[7] L. S. Finn and D. F. Chernoff, Phys. Rev. D 47, 2198 (1993).

[8] C. Cutler and E.E. Flanagan, Phys. Rev. D 49, 2658 (1994).

[9] E. S. Phinney, arXiv:astro-ph/0108028.

[10] A. J. Farmer and E. S. Phinney, Mon. Not. R. Astron. Soc. 346, 1197 (2003).

[11] S. Babak et al., Classical Quantum Gravity 25, 184026 (2008).

[12] C. Cutler and J. Harms, Phys. Rev. D 73, 042001 (2006).

[13] S. M. Rao, D. A. Turnshek, and D. B. Nestor, Astrophys. J. 636, 610 (2006).

[14] S. Perlmutter et al., Astrophys. J. 517, 565 (1999).

[15] D. N. Spergel et al., Astrophys. J. Suppl. Ser. 148, 175 (2003).

[16] A. M. Hopkins and J. Beacom, Astrophys. J. 651, 142 (2006).

[17] M. Fardal, N. Katz, D. Weinberg, and R. Davé, Mon. Not. R. Astron. Soc. 379, 985 (2007).

[18] S. M. Wilkins, N. Trentham, and A. Hopkins, Mon. Not. R. Astron. Soc. 385, 687 (2008).

[19] K. Nagamine, J. Ostriker, M. Fukugita, and R. Cen, Astrophys. J. 653, 881 (2006).

[20] T. Piran, Astrophys. J. 389, L45 (1992).

[21] S. Ando, J. Cosmol. Astropart. Phys. 06 (2004) 007.

[22] R. O’Shaughnessy, K. Belczynski, and V. Kalogera, Astrophys. J. 675, 566 (2008).

[23] J. A. de Freitas Pacheco, T. Regimbau, A. Spallici, and S. Vincent, Int. J. Mod. Phys. D 15, 235 (2006).

[24] A. V. Tutukov and L. R. Yungelson, Mon. Not. R. Astron. Soc. 268, 871 (1994).

[25] V. M. Lipunov, K. A. Postnov, M.E. Prokhorov, I.E. Panchenko, and H.E. Jorgensen, Astrophys. J. 454, 593 (1995).

[26] K. Belczynski, R. Perna, T. Bulik, V. Kalogera, N. Ivanova, and D. Q. Lamb, Astrophys. J. 648, 1110 (2006).
[27] E. Berger et al., Astrophys. J. 664, 1000 (2007).

[28] K. Belczynski and V. Kalogera, Astrophys. J. Lett. 550, L183 (2001).

[29] T. Bulik, K. Belczynski, and B. Rudak, Astron. Astrophys. 415, 407 (2004).

[30] E. S. Phinney, Astrophys. J. Lett. 380, L17 (1991).

[31] V. Kalogera, R. Narayan, D. N. Spergel, and J. H. Taylor, Astrophys. J. 556, 340 (2001).

[32] R. K. Kopparapu, C. R. Hanna, V. Kalogera, R. O'Shaughnessy, G. Gonzalez, P. R. Brady, and S. Fairhurst, Astrophys. J. 675, 1459 (2008).

[33] V. Kalogera et al., Astrophys. J. 614, L137 (2004).

[34] K. Postnov and L. R. Yungelson, Living Rev. Relativity 9, 6 (2006), http://www.livingreviews.org/lrr-2006-6.

[35] A. V. Tutukov and L. R. Yungelson, Mon. Not. R. Astron. Soc. 260, 675 (1993).

[36] R. Voss and T. M. Tauris, Mon. Not. R. Astron. Soc. 342, 1169 (2003).

[37] V.M. Lipunov, K. A. Postnov, and M.E. Prokhorov, Astron. Lett. 23, 492 (1997).

[38] S.F. Portegies Zwart and L. R. Yungelson, Astron. Astrophys. 332, 173 (1998).

[39] G. Nelemans, L. R. Yungelson, S. F. Portegies Zwart, and F. Verbunt, Astron. Astrophys. 365, 491 (2001).

[40] K. Belczynski, R. E. Taam, V. Kalogera, F. A. Rasio, and T. Bulik, Astrophys. J. 662, 504 (2007).

[41] R. O'Shaughnessy, C. Kim, V. Kalogera, and K. Belczynski, Astrophys. J. 672, 479 (2008).

[42] D. Coward and T. Regimbau, New Astron. Rev. 50, 461 (2006).

[43] S. Drasco and E. E. Flanagan, Phys. Rev. D 67, 082003 (2003).

[44] A. Lazzarini and R. Weiss, Report No. LIGO-E95001802, 1996.

[45] M. Punturo, Report No. VIR-NOT-PER-1390-51, 2004.

[46] R. Del Fabbro, A. Di Virgilio, A. Giazotto, H. Kautzky, V. Montelatici, and D. Passuello, Phys. Lett. A 124, 253 (1987).

[47] P. Fritschel, Second Generation Instruments for the Laser Interferometer Gravitational Wave Observatory, edited by M. Cruise and P. Saulson, SPIE Conference Series Vol. 4856, (2003), p. 282.

[48] R. Abbot et al., Report No. LIGO-T070247-01-I, 2008.

[49] Virgo Collaboration, Report No. VIR-089A-08, 2008. 